\title{
Initial Clinical Experience with Robotic Lateral Pelvic Lymph Node Dissection for Advanced Rectal Cancer
}

\author{
Ju-A Park, Gyu-Seog Choi, Jun Seok Park, Soo Yeun Park \\ Colorectal Cancer Center, Kyungpook National University Medical Center, Kyungpook National University School of Medicine, Daegu, Korea
}

Purpose: This study was conducted to evaluate the technical feasibility and safety of robotic extended lateral pelvic lymph node dissection (LPLD) in patients with advanced low rectal cancer.

Methods: A review of a prospectively-collected database at Kyungpook National University Medical Center from January 2011 to November revealed a series of 8 consecutive robotic LPLD cases with a preoperative diagnosis of lateral node metastasis. Data regarding patient demographics, operating time, perioperative blood loss, surgical morbidity, lateral lymph node status, and functional outcome were analyzed.

Results: In all eight patients, the procedures were completed without conversion to open surgery. The mean operative time of extended pelvic node dissection was 38 minutes (range, 20 to 51 minutes), the mean number of lateral lymph nodes harvested was 4.1 (range, 1 to 13), and 3 patients (38\%) were found to have lymph node metastases. Postoperative mortality and morbidity were $0 \%$ and $25 \%$, respectively, but, there was no LPLD-related morbidity. The mean hospital stay was 7.5 days (range, 5 to 12 days).

Conclusion: Robotic LPLD is safe and feasible, with the advantage of being a minimally invasive approach. Further largescale studies comparing robotic and conventional surgery with long-term follow-up evaluation are needed to confirm these findings.

\section{Keywords: Robotic surgery; Pelvic lymph node dissection; Rectal cancer}

\section{INTRODUCTION}

The presence of lateral pelvic (LP) lymph node metastasis in rectal cancer was first reported in the 1950s [1], but the clinical significance, management, and outcome of such cases remain controversial. Currently, lateral pelvic lymph node dissection (LPLD) is not regularly performed in Western countries because preoperative radiation followed by total mesorectal excision (TME) is the standard treatment for advanced rectal cancer [2-4]. However, a major study on LPLD based on the multiinstitutional data regis-

Received: August 31, 2012 - Accepted: October 23, 2012

Correspondence to: Gyu-Seog Choi, M.D.

Colorectal Cancer Center, Kyungpook National University Medical Center, Kyungpook National University School of Medicine, 807 Hoguk-ro, Buk-gu, Daegu 702-210, Korea

Tel: +82-53-200-2166, Fax: +82-53-200-2027

E-mail:kyuschoi@mail.knu.ac.kr

(c) 2012 The Korean Society of Coloproctology

This is an open-access article distributed under the terms of the Creative Commons Attribution NonCommercial License (http://creativecommons.org/licenses/by-nc/3.0) which permits unrestricted noncommercial use, distribution, and reproduction in any medium, provided the original work is properly cited. try in Japan found that LP lymph node metastasis (internal and external iliac) was present in $14.6 \%$ of patients with T3 or T4 tumors who had undergone LPLD [5]. Survival was significantly better in patients with LP lymph node metastasis than in stage-IV patients who had undergone a curative resection. These data supported the idea that LP lymph node metastasis could be considered as regional lymph node spreading rather than systemic metastasis.

The use of minimally invasive approaches is of growing interest for the treatment of rectal cancer. Several studies have shown that laparoscopic TME is associated with reduced surgical trauma and improved immediate postoperative outcomes, with a consequent reduction in both recovery times and periods of hospitalization. Nonetheless, very few authors have reported their experience with endo-laparoscopic TME with LPLD for advanced rectal cancer $[6$, 7]. In the narrow pelvis, this technique is difficult to master due in part to its technical limitations such as 2-dimensional views with reduced depth perception and poor ergonomics resulting in fatigue, shaking, and awkward positioning of surgeons.

Recently, robot-assisted surgery has emerged as an alternative minimally-invasive method. Given the difficult location of the LP 
node and the complex vascular structure, robot-assisted lymph node dissection was considered beneficial for the successful completion of LPLD. Beginning in 2008, a robotic surgical system was introduced into routine clinical practice at our institution. As our combined experience grew, we extended the indication for robotic colorectal surgery to almost all disease stages. Recently, we have begun to selectively perform robotic TME with LPLD for patients diagnosed with LP lymph node metastasis. In the present study, we report our initial experience with robotic TME in conjunction with LPLD for advanced rectal cancer.

\section{METHODS}

This study was approved by the local institutional review boards. A review of a prospectively collected database at Kyungpook National University Medical Center from January to November 2011 revealed a series of eight consecutive patients who underwent robotic LPLD for advanced rectal cancer. Data regarding patient demographics, operative results, surgical morbidity, and pathologic results, including lateral lymph node status, were reviewed.

Patients with suspected metastatic lymph nodes in the pelvic side wall based on radiological findings received long-course combined chemoradiation therapy (CCRT, 50 Gy in 25 fractions for 5 weeks and chemotherapy with 5 -fluorouracil/leucovorin) before surgical resection. In our institution, LPLD in addition to TME was performed when abnormal lymph nodes were identified in the pelvic side wall after preoperative radiation. Radiologicallypositive lymph node metastasis was diagnosed if a lymph node was enlarged by more than $5 \mathrm{~mm}$ or showed an abnormal configuration on computed tomography (CT) of the abdomen and on pelvic magnetic resonance imaging (MRI). Additional cycles of chemotherapy were administered after surgical resection. Each patient was given information regarding the surgical methods and procedures to be used, and written informed consent was obtained from all patients before surgery.

All procedures were performed by a single surgeon. Under general anesthesia, the patient was placed in a modified lithotomy position with both arms alongside the body. The procedures were performed under a 6-port system and $0^{\circ}$ endoscope: 1 camera port $(12 \mathrm{~mm}), 3$ ports $(8 \mathrm{~mm})$ for robot arms, and 2 ports $(5 \mathrm{~mm})$ for assist (Fig. 1). A hybrid technique was used, consisting of laparoscopic colonic mobilization with inferior mesenteric artery ligation and robotic TME and LPLD. After the laparoscopic procedure, the body of the robot system was introduced from the caudal side of the patient regardless of the site of pelvic lymph node dissection. The robot arms were docked facing the pelvic cavity. The dissection of lateral nodes commenced after the completion of TME and transection of the distal rectum. The first robot arm (with monopolar curved scissors) was used to perform the dissection. The second arm (with bipolar Cadiere forceps) was used to grasp and retract lymphoareolar tissue and was sometimes used as an energy device to control bleeding. The third arm (with dou-

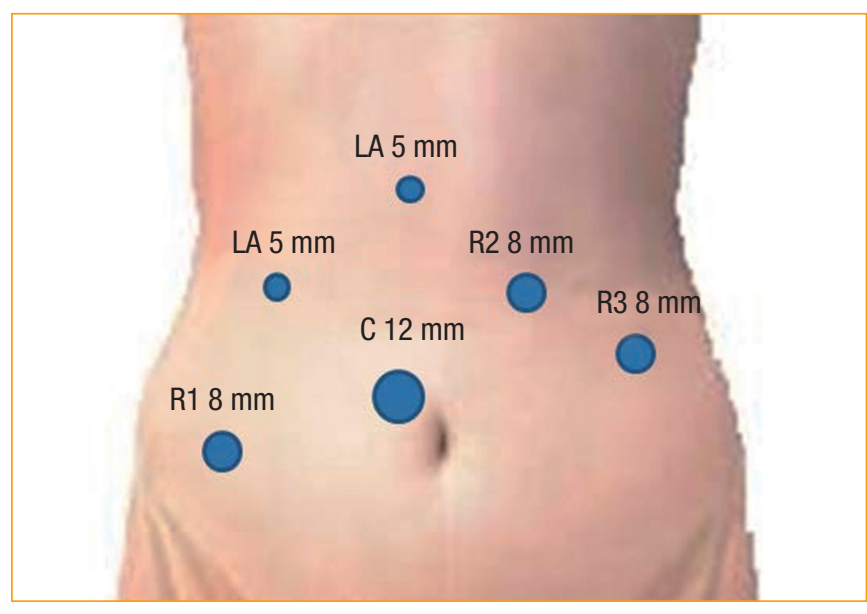

Fig. 1. Trocar placement in a robotic lateral pelvic lymph node dissection for advanced rectal cancer. LA, laparoscopic assistant; R, robot trocar; C, camera.

ble-fenestrated forceps) had the most important function in this procedure, as it facilitated the dissection by placing it for counterretraction. Additional retraction and fluid removal from the surgical field were provided by the assistant surgeon. This utilization of all robot arms was the same regardless of the site of LPLD.

LP nodes were categorized according to their location in one of three regions: the common iliac and external iliac region, the internal iliac region, and the obturator region. The dissection of these lymph nodes was initiated at the area between the common iliac bifurcation and the hypogastric nerve. The two tendon-like hypogastric nerves could be palpated just below the aortic bifurcation. While the parietal pelvic peritoneum over the area was elevated and pushed to the lateral side by the third robot arm, the monopolar scissors opened the peritoneum and dissected lymphoareolar tissue from the common iliac vessels. The dissection was extended downward and backward. The branches of the internal iliac artery and ureter were then exposed (Fig. 2A). The third arm gently pushed the external iliac vessels, ureter, and pelvic muscles towards the supero-lateral side in order to expose the branches of the internal iliac artery and obturator fossa. We flexed and opened the two limbs of the double fenestrated forceps to effectively perform this counter-retraction. The internal iliac vessels were then completely cleared from the lymphatic tissue down to the middle hemorrhoidal vessel. During this dissection, the obturator nerve and vessels were exposed above the obturator channel (Fig. 2B, C). At the anteromedial side of the internal iliac vessels is the facial layer bearing the hypogastric nerves and pelvic plexus. While the third arm provided the surgical field by counter-retracting the pelvic wall to expose dissection plane tenting, the second arm or assistant retracted the facial layer. The dissection of lymphoareolar tissues continued from the medial side of the fascial layer, with caution to prevent injury to nerve fibers (Fig. 2D). All lymphoareolar tissue in the obturator fossa was removed, leaving the obturator nerve and ves- 

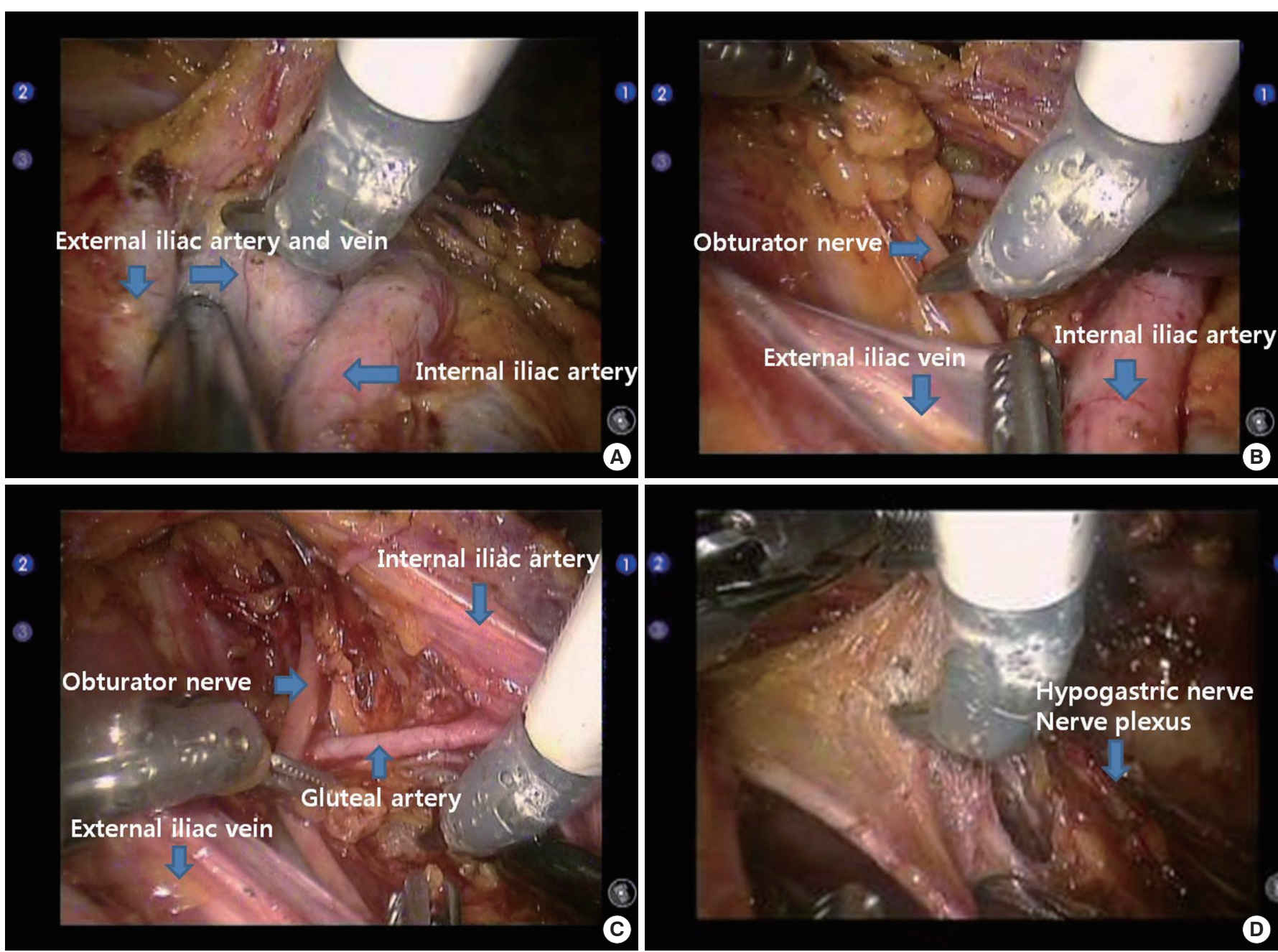

Fig. 2. Left-side lateral pelvic lymph node dissection for advanced rectal cancer using the robot surgical system. (A) Dissection of a lymph node around the external iliac artery and internal iliac artery. (B-D) Isolation of the obturator nerve and vessels above the obturator channel. Removal of the lymphoareolar tissue in the obturator fossa, exposing the obturator nerve and vessel.

sel bare. When positive lymph nodes were found or suspected in the lateral lymphatic channels around the internal iliac vessel and its branches, we carefully skeletonized the vessels and nerves. However, when the metastatic lymph nodes encased them, they were sacrificed for en-block resection. The lymph nodes were collected in a plastic bag and retrieved along with the main specimen [8].

\section{RESULTS}

Demographic and intraoperative data are detailed in Table 1. Enrolled patients included 3 women and 5 men, aged from 45 to 85 years, all of whom had rectal cancer that had spread to the LP lymph nodes at initial radiologic staging. All these patients underwent preoperative CCRT, followed by LPLD, which was bilateral in two patients and unilateral in six patients. Sphincter-preserving rectal cancer surgery was performed in each case and consisted of an intersphincteric resection with coloanal anastomosis for six patients and a low anterior resection for the remaining two patients. Fecal diversion was performed for five patients to protect the anastomosis. In all eight patients, the procedures were completed without the need for conversion to laparoscopic or open surgery. The median operation time from skin incision to closure was $273 \mathrm{~min}$ utes (range, 170 to 350 minutes). The median time for LPLD of one side of the pelvic wall was 38 minutes (range, 20 to 51 minutes). The median blood loss was $48 \mathrm{~mL}$ (range, 20 to $100 \mathrm{~mL}$ ). None of the patients needed a blood transfusion during the perioperative period, and the hypogastric nerve, pelvic nerve plexus, and obturator nerve were identified and preserved in all patients.

Postoperative outcomes and pathologic results are summarized in Table 2. No operative mortality was noted. Postoperative morbidity developed in two patients without LPLD-related morbidity. One patient (case 2) required a repeat operation for fecal diversion 
Table 1. Patient characteristics and operative findings

\begin{tabular}{lcccccccccc}
\hline Case no. & Age & Sex & BMl $\left(\mathrm{kg} / \mathrm{m}^{2}\right)$ & ASA class & $\begin{array}{c}\text { Tumor height } \\
(\mathrm{cm})\end{array}$ & $\begin{array}{c}\text { Preop. } \\
\text { CCRT }\end{array}$ & $\begin{array}{c}\text { Surgical } \\
\text { procedure }\end{array}$ & $\begin{array}{c}\text { Total OP } \\
\text { time }(\mathrm{min})\end{array}$ & $\begin{array}{c}\text { Time for } \\
\text { LPLD (min) }\end{array}$ & $\begin{array}{c}\text { Blood loss } \\
(\mathrm{mL})\end{array}$ \\
\hline 1 & 61 & F & 21.2 & 1 & 5.0 & Yes & ISR/both & 350 & 51 & 50 \\
2 & 85 & F & 21.2 & 2 & 3.0 & Yes & ISR/left & 220 & 25 & 20 \\
3 & 58 & M & 27.6 & 2 & 4.0 & Yes & ISR/left & 260 & 27 & 30 \\
4 & 47 & M & 22.8 & 1 & 4.0 & Yes & ISR/both & 330 & 41 & 50 \\
5 & 51 & M & 25.8 & 1 & 7.0 & Yes & LAR/left & 240 & 47 & 80 \\
6 & 71 & F & 24.1 & 2 & 4.0 & Yes & LAR/left & 300 & 43 & 100 \\
7 & 58 & M & 16.2 & 2 & 3.5 & Yes & ISR/left & 310 & 47 & 20 \\
8 & 45 & M & 20.6 & 1 & 2.0 & Yes & ISR/left & 170 & 20 \\
\hline
\end{tabular}

BMI, body mass index; ASA, American Society of Anesthesiologists; Preop. CCRT, preoperative concurrent chemoradiation therapy; OP, operative; LPLD, lateral pelvic lymph node dissection; ISR, intersphincteric resection; LAR, low anterior resection.

Table 2. Postoperative and pathologic outcomes

\begin{tabular}{|c|c|c|c|c|c|c|c|}
\hline Case no. & $\begin{array}{l}\text { Hospital stay } \\
\text { (day) }\end{array}$ & $\begin{array}{c}\text { Time to regular } \\
\text { diet (day) }\end{array}$ & Complication & $\begin{array}{c}\text { No. of lymph } \\
\text { nodes harvested }\end{array}$ & $\begin{array}{l}\text { No. of retrieved } \\
\text { LPLN }\end{array}$ & $\begin{array}{l}\text { No. of positive } \\
\text { LPLN }\end{array}$ & ypTNM $^{a}$ \\
\hline 1 & 10 & 8 & - & 7 & 5 & 0 & T1N0 \\
\hline 2 & 8 & 5 & Anastomotic leakage & 11 & 1 & 0 & T3NO \\
\hline 3 & 5 & 5 & - & 5 & 3 & 1 & T2NO \\
\hline 4 & 7 & 4 & - & 21 & 13 & 0 & TONO \\
\hline 5 & 6 & 5 & Trans luminal bleeding & 6 & 1 & 1 & T3N1 \\
\hline 6 & 7 & 4 & - & 15 & 5 & 1 & T4N2 \\
\hline 7 & 12 & 10 & - & 11 & 4 & 0 & T3N1a \\
\hline 8 & 5 & 4 & - & 5 & 1 & 0 & T3NO \\
\hline
\end{tabular}

LPLN, lateral pelvic lymph node; TNM, tumor-node-metastasis.

${ }^{a}$ According to the seventh edition of the American Joint Committee on Cancer cancer staging.

because of anastomotic leakage. Transluminal bleeding developed in case 6 and was controlled by performing a transanal suture. No patient experienced complications associated with LPLD. For all patients, the urinary catheter was removed at postoperative day 2 to 3 , and none of them suffered short- or long-term urinary problems. The median hospital stay was 7.5 days (range, 5 to 12 days). Complete remission of the tumor after CCRT was found in $1 \mathrm{pa}-$ tient (12.5\%, case 4$)$, and this patient had no positive lymph nodes amongst the total of 13 pelvic lymph nodes dissected from both sides. The total number of harvested lymph nodes, including pelvic lymph nodes, ranged from 5 to 21 , the median number of harvested lateral lymph nodes was 4.1 (range, 1 to 13 ), and positive lymph nodes were identified in 3 patients (38\%). One patient (case 3) had no positive regional lymph nodes from the mesorectum, but did have 1 positive lymph node from LPLD.

\section{DISCUSSION}

Different treatment strategies have evolved in Eastern and Western countries to reduce the local recurrence rates of rectal cancer.
In most Western institutions, neoadjuvant therapy, especially preoperative radiation therapy, is most commonly employed in conjunction with TME. Many surgeons in Western countries are still skeptical about the value of LPLD for the treatment of advanced low rectal cancers because en bloc lymph node dissection with pelvic autonomic nerve preservation is technically challenging. In the East, LP nodal involvement has been regarded as a factor associated with a poor prognosis, increased incidence of local recurrence, and reduced survival $[2,9,10]$. Subsequently, a growing body of literature, mostly from Japanese institutions, supported the use of an extended pelvic lymphadenectomy for cases of advanced low rectal tumors. The incidence of local recurrence in patients with rectal cancer who undergo TME without LPLD in Western countries is reported to be less than 10\% [11-14]. Although this incidence is similar to that for patients undergoing TME with LPLD, a direct comparison is difficult because of differences in clinical backgrounds.

Only a few studies have reported the technical feasibility of laparoscopic LPLD with TME for rectal cancer $[6,7,15]$. We previously reported 16 cases of laparoscopic LPLD for low rectal can- 
cer after concurrent chemoradiation [8]. All procedures were successfully performed without conversion to open surgery, and the mean blood loss and operative time were $190 \mathrm{~mL}$ and $310 \mathrm{~min}$ utes, respectively. Importantly, no severe urinary or sexual morbidity was observed in any of these patients. Although we have conducted laparoscopic LPLD in the past, it is difficult to be certain whether the robotic approach has provided clinical benefits over LPLD performed using conventional laparoscopy. We report only on the safety and the feasibility of robotic LPLD in this study because a comparative analysis would be flawed because of unmatched variables and a limited number of cases. Nevertheless, we have observed that lymph node dissection around the dedicated internal iliac vessel was easier when using a robotic interface.

Compared with conventional laparoscopy, one potential advantage of robotic LPLD may be the improved surgical view owing to 3-dimensional imaging and a surgeon-controlled camera platform. In addition, the surgeon can operate with two hands, using one, the assistant, to provide adequate exposure, compared to having just one hand for dissection, and the other for exposure, as is the case for conventional laparoscopy. The main vessel and its tributaries caused little difficulty during the dissection.

With the exception of surgeons in Japan, most surgeons do not use this procedure. Problems associated with LPLD, compared to typical conventional surgery, include urinary and sexual dysfunction and a longer operating time with greater blood loss. Recently, Georgiou et al. [15] used a meta-analysis to evaluate the impact of an extended lymphadenectomy in treating rectal cancer and found that the extended lymphadenectomy did not confer a cancer-specific survival advantage and was associated with an increased incidence of complications (urinary and/or sexual dysfunction). Moriya et al. [16] reported a mean operating time and blood loss of 393 minutes and 2,128 mL, respectively, for 53 patients undergoing LPLD with internal iliac vessel excision. Even center-of-excellence data on performing a LP lymphadenectomy showed a discouraging 5-year survival rate of $42 \%$ in patients with a positive lymph node and complete genitourinary functional loss in $33 \%$ of patients 2 years after LP node dissection [4, 17]. In our series, the median blood loss was $48 \mathrm{~mL}$, and the median operation time was 273 minutes, calculated separately from the standardized TME procedure. Remarkably, none of the patients had accidental massive blood loss or conversion to open surgery. The postoperative mortality and morbidity rates were $0 \%$ and $25 \%$, respectively, and no LPLD-related morbidity, such as lymphoceles and urinary dysfunction, were noted. The present study also showed rapid postoperative recovery, with a median hospital stay of 7.5 days, similar to that reported for most case series of laparoscopic colorectal surgery [6]. We believe that the increased maneuverability of the instruments with a robotic system is the most likely reason for the short operation time and the reduced morbidity. In our center, robotic surgery is currently becoming central to the treatment strategy for patients with pelvic lymph metastasis.

As mentioned above, routine adoption of LPLD for patients with advanced rectal cancer is controversial because less than $15 \%$ of patients with locally advanced rectal cancer have lateral lymph node metastasis. Therefore, we adopt a more discerning policy in the selection of patients for LPLD, excluding patients with extraperitoneal rectal cancer when no radiologically-suspicious lymph nodes are identified. When radiologically-lateral lymph node metastasis is suspected and the patient's tumor is located in an extraperitoneal region, usually preoperative chemoradiation is performed first. TME is provided if lateral lymph node metastasis is not found after chemoradiation or if the tumor appears to be clinically benign. Conversely, even after preoperative chemoradiation, LPLD is performed when lateral node metastasis is suspected on MRI or positron emission tomography/CT. Under such selective conditions, the positive rate of lateral lymph node metastasis was $38 \%, 1.5$ times higher than that previously reported in Japan. We expect the frequency of unnecessary LPLDs to be reduced if more sensitive radiologic criteria are established.

The current study has inherent limitations. It is retrospective in nature and has a small sample size. Functional and oncological outcomes are not sufficient, and the follow-up period is limited. Despite these limitations, this is the first report on a series of patients treated with robot-assisted LPLD following TME that documents the feasibility of this procedure. Robotic LPLD seems to have excellent short-term surgical and pathological outcomes and satisfactory functional results. Large-scale studies are needed to evaluate potential differences in the outcomes between conventional open, laparoscopy, and robot-assisted LPLD.

\section{CONFLICT OF INTEREST}

No potential conflict of interest relevant to this article was reported.

\section{ACKNOWLEDGMENTS}

This research was supported by the Kyungpook National University research fund 2009 (No.00000000_09-1008).

\section{REFERENCES}

1. Sauer I, Bacon HE. Influence of lateral spread of cancer of the rectum on radicability of operation and prognosis. Am J Surg 1951; 81:111-20.

2. Ueno H, Mochizuki H, Hashiguchi Y, Hase K. Prognostic determinants of patients with lateral nodal involvement by rectal cancer. Ann Surg 2001;234:190-7.

3. Fujita S, Yamamoto S, Akasu T, Moriya Y. Lateral pelvic lymph node dissection for advanced lower rectal cancer. Br J Surg 2003; 90:1580-5.

4. Moriya Y, Sugihara K, Akasu T, Fujita S. Importance of extended lymphadenectomy with lateral node dissection for advanced lower rectal cancer. World J Surg 1997;21:728-32.

5. Akiyoshi T, Watanabe T, Miyata S, Kotake K, Muto T, Sugihara K, 
et al. Results of a Japanese nationwide multi-institutional study on lateral pelvic lymph node metastasis in low rectal cancer: is it regional or distant disease? Ann Surg 2012;255:1129-34.

6. Liang JT. Technical feasibility of laparoscopic lateral pelvic lymph node dissection for patients with low rectal cancer after concurrent chemoradiation therapy. Ann Surg Oncol 2011;18:153-9.

7. Konishi T, Kuroyanagi H, Oya M, Ueno M, Fujimoto Y, Akiyoshi T, et al. Multimedia article. Lateral lymph node dissection with preoperative chemoradiation for locally advanced lower rectal cancer through a laparoscopic approach. Surg Endosc 2011;25: 2358-9.

8. Park JS, Choi GS, Lim KH, Jang YS, Kim HJ, Park SY, et al. Laparoscopic extended lateral pelvic node dissection following total mesorectal excision for advanced rectal cancer: initial clinical experience. Surg Endosc 2011;25:3322-9.

9. Kusters M, Beets GL, van de Velde CJ, Beets-Tan RG, Marijnen CA, Rutten HJ, et al. A comparison between the treatment of low rectal cancer in Japan and the Netherlands, focusing on the patterns of local recurrence. Ann Surg 2009;249:229-35.

10. Yano H, Moran BJ. The incidence of lateral pelvic side-wall nodal involvement in low rectal cancer may be similar in Japan and the West. Br J Surg 2008;95:33-49.
11. Enker WE, Thaler HT, Cranor ML, Polyak T. Total mesorectal excision in the operative treatment of carcinoma of the rectum. J Am Coll Surg 1995;181:335-46.

12. Heald RJ, Moran BJ, Ryall RD, Sexton R, MacFarlane JK. Rectal cancer: the Basingstoke experience of total mesorectal excision, 1978-1997. Arch Surg 1998;133:894-9.

13. Lopez-Kostner F, Lavery IC, Hool GR, Rybicki LA, Fazio VW. Total mesorectal excision is not necessary for cancers of the upper rectum. Surgery 1998;124:612-7.

14. Zaheer S, Pemberton JH, Farouk R, Dozois RR, Wolff BG, Ilstrup D. Surgical treatment of adenocarcinoma of the rectum. Ann Surg 1998;227:800-11.

15. Georgiou P, Tan E, Gouvas N, Antoniou A, Brown G, Nicholls RJ, et al. Extended lymphadenectomy versus conventional surgery for rectal cancer: a meta-analysis. Lancet Oncol 2009;10:1053-62.

16. Moriya Y, Hojo K, Sawada T, Koyama Y. Significance of lateral node dissection for advanced rectal carcinoma at or below the peritoneal reflection. Dis Colon Rectum 1989;32:307-15.

17. Ueno M, Oya M, Azekura K, Yamaguchi T, Muto T. Incidence and prognostic significance of lateral lymph node metastasis in patients with advanced low rectal cancer. Br J Surg 2005;92:756-63. 